\title{
Receding-horizon adaptive control of aero-optical wavefronts
}

\author{
Jonathan Tesch \\ Jet Propulsion Laboratory \\ Wavefront Sensing and Control \\ Pasadena, California 91109 \\ E-mail: Jonathan.A.Tesch@jpl.nasa.gov

\section{Steve Gibson} \\ University of California \\ Mechanical and Aerospace Engineering \\ Los Angeles, California 90095-1597 \\ E-mail: gibson@ucla.edu

\section{Michel Verhaegen} \\ Delft University of Technology \\ Delft Center for Systems and Control \\ Delft 2628 CD, The Netherlands
}

\begin{abstract}
A new method for adaptive prediction and correction of wavefront errors in adaptive optics $(A O)$ is introduced. The new method is based on receding-horizon control design and an adaptive lattice filter. Experimental results presented illustrate the capability of the new adaptive controller to predict and correct aero-optical wavefronts derived from recent flight-test data. The experimental results compare the performance of the new adaptive controller the performance of a minimum-variance adaptive controller previously used in AO. These results demonstrate the reduced sensitivity of the receding-horizon adaptive controller to high-frequency sensor noise. () 2013 Society of Photo-Optical Instrumentation Engineers (SPIE) [DOI: 10.1117/1.OE.52.7.071406]
\end{abstract}

Subject terms: adaptive control; adaptive optics; adaptive filtering; aero-optics.

Paper 121433SSP received Oct. 1, 2012; revised manuscript received Dec. 18, 2012; accepted for publication Jan. 2, 2013; published online Mar. 22, 2013.

\section{Introduction}

Improved wavefront correction in adaptive optics (AO) has been achieved by adaptive filtering and control ${ }^{1-5}$ and by optimal linear time-invariant (LTI) filtering and control. ${ }^{6-17}$ Both approaches extend the limited bandwidth of classical AO control loops by predicting wavefront aberrations to mitigate loop latency. This paper introduces an adaptive controller designed to minimize a receding-horizon performance index. Results from an $\mathrm{AO}$ experiment are used to compare the performance of the new adaptive controller to the performance of the minimum-variance adaptive controller previously used for $\mathrm{AO} .^{4,5,17}$ In the experiment, aero-optical wavefronts measured in the University of Notre Dame's Airborne Aero-Optics Laboratory (AAOL) ${ }^{18}$ were mapped to the geometry of the University of California Los Angeles (UCLA) AO experiment and added to the laser beam to provide wavefront disturbance.

Receding-horizon control, also known as generalized predictive control (GPC), ${ }^{19-21}$ provides a large design space that can incorporate frequency weighting and uncertainty models to improve robustness with respect to sensor noise and plant modeling error. Also, receding-horizon adaptive control can accommodate coupled control channels and deformable mirror dynamics better than the adaptive controllers previously used for AO can. This paper demonstrates the effectiveness of frequency weighting for making the receding-horizon adaptive controller robust to high-frequency wavefront sensor noise. A form of frequency weighting has been used previously to reduce sensitivity to sensor noise in minimumvariance adaptive control of tilt jitter, ${ }^{22}$ but the recedinghorizon approach introduced here allows frequency weighting to be incorporated into the adaptive control design in a more direct and flexible way. The experimental results in this paper illustrate the capability of the receding-horizon adaptive controller with frequency weighting to avoid amplifying high-frequency noise added to the wavefront sensor

0091-3286/2013/\$25.00 @ 2013 SPIE measurements, whereas the minimum-variance adaptive controller used for comparison amplifies the effect of the sensor noise on the closed-loop wavefronts.

A class of minimum-variance adaptive controllers used previously for $\mathrm{AO}^{4,5,17}$ and control of tilt jitter ${ }^{22,23}$ were based on multichannel recursive least-squares (RLS) lattice filters that predicted the disturbance one or two time steps ahead. The adaptive controller introduced here also uses a RLS lattice filter for adaptive prediction, but the quantity predicted in this paper is quite different from the disturbance signals predicted previously. Here, the adaptive filter predicts a certain linear combination of the disturbance sequence over the receding horizon. This linear combination of future disturbances is determined by the plant model and the recedinghorizon performance index. The use of the adaptive lattice filter to predict the required quantity for receding-horizon control and how the adaptive filter is integrated into the controller are the novel aspects of the adaptive controller presented here.

Section 2 presents the receding-horizon adaptive controller. Section 3 describes the adative optics experiment and the aerooptical wavefronts used as the disturbance. Section 4 describes the control model of the experiment and the classical AO control loop, which the adaptive control loop augments. Section 5 presents the experimental results comparing the performance of the receding-horizon adaptive controller, the lattice-filter based minimum-variance adaptive controller used previously for $\mathrm{AO}^{4,5,17}$ and the classical $\mathrm{AO}$ loop alone.

\section{Receding-Horizon Adaptive Control}

\subsection{Receding-Horizon Control Law}

The linear-quadratic receding-horizon control problem in this paper is based on the following state-space model of the plant:

$$
\begin{aligned}
& x_{p}(t+1)=A_{p} x_{p}(t)+B_{p} u(t), \\
& e(t)=C_{p} x_{p}(t)+w(t),
\end{aligned}
$$


where $t(=0,1,2, \ldots)$ is the sample time or sample number, $x_{p}$ is the state vector, $e$ is the measured output vector and $w$ is output disturbance. This plant model is assumed to be stable. The model may represent either an open-loop plant or a closed-loop plant consisting of an open-loop plant with a stabilizing feedback controller. In the latter case, $w$ represents residual output disturbance; i.e., output disturbance not corrected by the feedback controller. The purpose of the adaptive controller presented here is to minimize the output error due to $w$. In $\mathrm{AO}$, the signal $w$ represents the combined effects of optical wavefront disturbance and wavefront sensor noise, which is a primary concern in this paper.

Since only the sequence $e$ is measured in applications, estimates $\hat{x}(t)$ and $\hat{w}(t)$ are used instead of $x(t)$ and $w(t)$. Also, only an estimate of the plant is available in applications. Hence, in the receding-horizon control structure presented here, the plant model in Eqs. (1) and (2) is replaced by

$\hat{x}(t+1)=\hat{A} \hat{x}(t)+\hat{B} u(t)$

$e(t)=\hat{C} \hat{x}(t)+\hat{w}(t)$

For fixed finite horizon length $h$, the column vectors $w_{h}(t)$ and $u_{h}(t)$ are defined (with standard MATLAB notation) by

$\hat{w}_{h}(t)=[\hat{w}(t+1) ; \hat{w}(t+2) ; \ldots \hat{w}(t+h)]$,

$u_{h}(t)=[u(t) ; u(t+1) ; \ldots u(t+h-1)]$.

The quadratic performance index used in this paper is

$$
\begin{aligned}
J_{h}\left[\hat{x}(t), \hat{w}_{h}(t), u_{h}(t)\right]= & \left\{\sum _ { k = 1 } ^ { h } \left[e^{T}(t+k) Q_{1} e(t+k)\right.\right. \\
& \left.+\hat{x}^{T}(t+k) Q_{2} \hat{x}(t+k)\right] \\
& \left.+\sum_{k=0}^{h-1}\left[u^{T}(t+k) R u(t+k)\right]\right\} \\
& +\hat{x}^{T}(t+h) Q_{3} \hat{x}(t+h)
\end{aligned}
$$

with the matrices $Q_{i}$ symmetric and positive semi-definite and the matrix $R$ symmetric and positive definite. The performance index in Eq. (6) can be written as

$$
\begin{aligned}
J_{h}\left[\hat{x}(t), \hat{w}_{h}(t), u_{h}(t)\right]= & \left\{\left[\begin{array}{l}
\hat{x}(t) \\
\hat{w}_{h}(t)
\end{array}\right]+\tilde{B} u_{h}(t)\right\}^{T} \\
& \times \tilde{Q}\left\{\left[\begin{array}{l}
\hat{x}(t) \\
\hat{w}_{h}(t)
\end{array}\right]+\tilde{B} u_{h}(t)\right\} \\
& +u_{h}(t)^{T} \tilde{R} u_{h}(t)
\end{aligned}
$$

with the matrices $\tilde{Q}, \tilde{R}$, and $\tilde{B}$ derived easily in terms of the matrices in Eqs. (3), (4), and (6). For the experimental results in this paper, the horizon length was $h=20$. Larger horizon lengths did not improve the performance of the adaptive controller, but the optimal horizon length will depend on plant dynamics and disturbance statistics in particular applications.

The receding-horizon control command $u(t)$ is chosen to be the first term in the vector $u_{h}(t)$ that minimizes $J_{h}\left[\hat{x}(t), \hat{w}_{h}(t), u_{h}(t)\right]$. Since the minimizing $u_{h}(t)$ is a linear

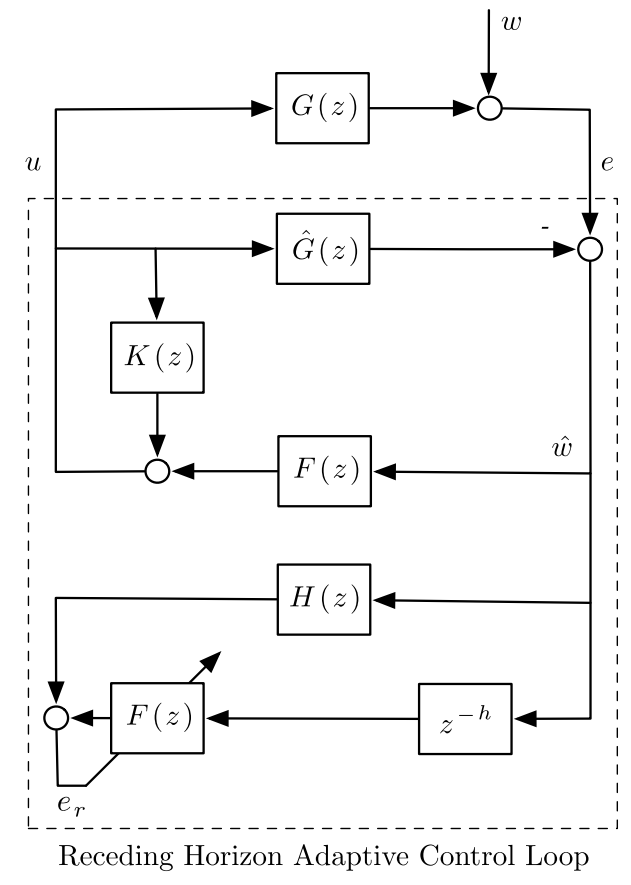

Fig. 1 Block diagram for receding-horizon adaptive control.

function of $\hat{x}(t)$ and $\hat{w}_{h}(t)$, the receding-horizon control command can be written

$u(t)=-K_{x} \hat{x}(t)-H_{w} \hat{w}_{h}(t)$,

where $K_{x}$ and $H_{w}$ are matrices derived easily in terms of $\tilde{Q}$, $\tilde{R}$, and $\tilde{B}$. It should be noted that at each sample time $t$, the vector $H_{w} \hat{w}_{h}(t)$ is a linear combination of the $h$ future values of $\hat{w}$ in Eq. (5).

The block diagram in Fig. 1 shows the receding-horizon adaptive controller inside the dashed box. In Fig. $1, G(z)$ is the transfer function from $u$ to $e$ for the plant in Eqs. (1) and (2), and $\hat{G}(z)$ is the transfer function from $u$ to $e$ for the plant model in Eqs. (3) and (4). Hence $\hat{G}(z)$ has the statespace realization

$\hat{G}(z)=(\hat{A}, \hat{B}, \hat{C}, 0)$.

As shown in Fig. 1, the adaptive controller constructs the signal $\hat{w}$ as

$\hat{w}=e-\hat{G}(z) u=w+[G(z)-\hat{G}(z)] u$.

The output of the $K(z)$ block in Fig. 1 is the term $-K_{x} \hat{x}$ in Eq. (8). It follows from Eqs. (3) and (8) that $K(z)$ has the state-space realization

$K(z)=\left(\hat{A}, \hat{B},-K_{x}, 0\right)$.

The adaptive part of the controller is the adaptive filter $F(z)$, which generates an estimate of the signal $-H_{w} \hat{w}_{h}$ in Eq. (8). The signal $H_{w} \hat{w}_{h}$ can be written as

$H_{w} \hat{w}_{h}=z^{h} H(z) \hat{w}$

where $H(z)$ is a finite-impulse-response (FIR) filter with $h$ taps; i.e., 


$$
H(z)=\sum_{k=0}^{h-1} H_{k} z^{-k},
$$

where the $H_{k}$ are gains (matrix gains, in general). Since Eq. (12) is equivalent to

$H(z) \hat{w}=z^{-h} H_{w} \hat{w}_{h}$

the output of the $H(z)$ block in Fig. 1 is the signal $H_{w} \hat{w}_{h}$ delayed by $h$ time steps.

Figure 1 shows that the adaptive control command signal satisfies

$u=[I-K(z)]^{-1} F(z) \hat{w}$.

It follows from Eq. (11) that $[I-K(z)]^{-1}$ has the state-space realization

$[I-K(z)]^{-1}=\left(\hat{A}-\hat{B} K_{x}, \hat{B},-K_{x}, I\right)$

Hence, the weighting matrices in Eq. (6) must be chosen so that the resulting gain matrix $K_{x}$ makes $\hat{A}-\hat{B} K_{x}$ stable. A common way to guarantee that $\hat{A}-\hat{B} K_{x}$ is stable is to choose the matrix $Q_{3}$ in Eq. (6) so that

$$
\hat{C}^{T} Q_{1} \hat{C}+Q_{2}+Q_{3}=P
$$

where the symmetric matrix $P$ is a stabilizing solution to the algebraic Riccati equation

$$
P=\hat{C}^{T} Q_{1} \hat{C}+Q_{2}+\hat{A}^{T}\left[P-P \hat{B}\left(R+\hat{B}^{T} P \hat{B}\right)^{-1} \hat{B}^{T} P\right] \hat{A} .
$$

In this case, the gain matrix $K_{x}$ is

$$
K_{x}=\left(R+\hat{B}^{T} P \hat{B}\right)^{-1} \hat{B}^{T} P \hat{A} .
$$

If $Q_{3}$ is not chosen to satisfy Eq. (17), $K_{x}$ still has the form in Eq. (19) with $P$ generated by the Riccati difference equation from the finite-time linear-quadratic optimal regulator (LQR) problem. ${ }^{21}$ The Riccati matrix, or sequence of Riccati matrices if Eq. (17) does not hold, can be used in a recursive construction of $H_{w}$. Alternatively, $H_{w}$ and $K_{x}$ can be computed by writing the $u_{h}(t)$ that minimizes the right side of Eq. (7) in terms of $\tilde{Q}, \tilde{R}, \tilde{B}, \hat{x}(t)$, and $\hat{w}_{h}(t)$.

It should be noted that the receding-horizon adaptive controller requires no disturbance model, either a priori or identified adaptively. However, a priori estimates of either the spectrum of the disturbance or the sensor noise (as in Sec. 5) can be used to determine frequency-weighting filters.

Several straightforward generalizations and variations of the receding-horizon adaptive controller presented here are possible. For example, the performance index in Eq. (6) can be generalized to obtain a linear-quadratic minimax problem to make the controller more robust with respect to plant modeling error, state estimator such as a Kalman filter can be used instead of the plant model in Eqs. (3) and (4) to generate $\hat{x}$, and different horizon lengths can be used for the various terms penalized in the performance index.

\subsection{Adaptive Filter}

The adaptive filter $F(z)$ estimates $-H_{w} \hat{w}_{h}(t)$ from data available at sample time $t$. The controller contains two copies of $F(z)$ with the same gains. The top copy of $F(z)$ in Fig. 1 generates the estimate of $-H_{w} \hat{w}_{h}(t)$ used in the control law, while the gains in $F(z)$ are updated adaptively in the copy of $F(z)$ in the bottom of Fig. 1.

The construction of the signal $\hat{w}$ in Eq. (10) provides the current $\hat{w}(t)$ at each sample time $t$, whereas $H_{w} \hat{w}_{h}(t)$ involves $\hat{w}(t+k)(k=1,2, \ldots, h)$. In principle, an adaptive prediction filter could be used to predict these $h$ future values of $\hat{w}$; however, the adaptive controller presented here takes the more efficient approach of using $F(z)$ to predict only the particular linear combination of future values of $\hat{w}$ required for the control law in Eq. (8). As indicated in the bottom part of Fig. 1, the adaptive filter is tuned to minimize the variance of the signal

$e_{r}=H(z) \hat{w}+F(z) z^{-k} \hat{w}$.

Since this is equivalent to minimizing the variance of $z^{k} H(z) \hat{w}+F(z) \hat{w}$, the top copy of $F(z)$ in Fig. 1 generates a minimum-variance prediction of $-z^{k} H(z) \hat{w}=-H_{w} \hat{w}_{h}$.

The adaptive filter used here is a multichannel RLS lattice filter ${ }^{24}$ with finite-impulse-response. In principle, a transversal FIR filter could be used in the adaptive controller, along with any multichannel RLS algorithm for updating the filter gains, including the classical RLS algorithm. ${ }^{25}$ The filter $H(z)$ in the adaptive controller has the standard transversal FIR form in Eq. (13), but the filter $F(z)$ has a more complex lattice filter realization. ${ }^{24}$ The particular lattice filter and associated RLS updating algorithm used here possess superior numerical stability and efficiency due to orthogonalization of the data channels. ${ }^{24}$ This is particularly important in AO applications due to the large number of inputs and outputs.

The lattice filter used for the experiments reported in this paper had fixed order $N=9$ (i.e., 10 taps) and 15 channels. There is no apparent relationship between the horizon length $h$ in the receding-horizon performance index and the order $N$ of the adaptive prediction filter $F(z)$. Normally, both $h$ and $N$ will be chosen large enough that larger orders yield no significant performance improvements.

\subsection{Frequency Weighting}

The receding-horizon performance index in Eq. (6) provides a large control design space parameterized by the weighting matrices $Q_{i}$ and $R$. Also, like other control schemes based on linear-quadratic optimization, receding-horizon adaptive control provides a convenient way to introduce frequency weighting by expanding the state vector and plant model to include state-space realizations of weighting filters applied to the control signal and/or the output error. This has been noted previously. ${ }^{19}$ For the experiment reported in this paper, a high-pass weighting filter was applied to the control signal to prevent the controller from amplifying high-frequency sensor noise. Since the only signals available to the adaptive controller are the output error vector $e$ and the control signal $u$, the adaptive controller has no way to distinguish between wavefront disturbance, which should be corrected, and sensor noise, which should not be passed through to the wavefront by the controller. But if an approximate bandwidth for 
sensor noise is known, frequency weighting in the control design can prevent the adaptive controller from putting a significant amount of the noise on the wavefront, as demonstrated in Sec. 5.

The procedure for applying frequency weighting to the adaptive control signal $u$ will be described now, since this is type of frequency weighting used in the AO application in this paper. A similar procedure can be used to apply frequency weighting to the output error signal $e$.

Any finite-dimensional LTI weighting filter in state-space form can be incorporated in the receding-horizon design, but since a delay in the weighting filter makes little or no difference in the performance of the controller, the weighting filter is assumed to have the state-space realization $\left(A_{f}, B_{f}, C_{f}, 0\right)$. The state vector $\hat{x}$ in Eqs. (3)-(8) has the form

$\hat{x}=\left[\begin{array}{l}\hat{x}_{p} \\ x_{f}\end{array}\right]$

where $\hat{x}_{p}$ is the estimate of the plant state vector in Eqs. (1) and (2) and $x_{f}$ is the state vector in the weighting filter. The matices $\hat{A}, \hat{B}$, and $\hat{C}$ in Eqs. (3) and (4) and the matrix $Q_{2}$ in Eq. (6) have the forms

$$
\begin{aligned}
& \hat{A}=\left[\begin{array}{cc}
\hat{A}_{p} & 0 \\
0 & A_{f}
\end{array}\right], \quad \hat{B}=\left[\begin{array}{l}
\hat{B}_{p} \\
B_{f}
\end{array}\right], \quad \hat{C}=\left[\begin{array}{ll}
\hat{C}_{p} & 0
\end{array}\right], \\
& Q_{2}=\left[\begin{array}{cc}
0 & 0 \\
0 & C_{f}^{T} C_{f}
\end{array}\right] .
\end{aligned}
$$

With $\hat{A}, \hat{B}$ and $\hat{C}$ having the forms in Eq. (22), the state-space realization of $\hat{G}(z)$ in Eq. (9) reduces to

$$
\hat{G}(z)=\left(\hat{A}_{p}, \hat{B}_{p}, \hat{C}_{p}, 0\right),
$$

which is an estimate of the plant model in Eqs. (1) and (2).

\section{Adaptive Optics Experiment}

\subsection{Experimental Layout}

The experiment, shown in the photograph in Fig. 2, has the optical layout in Fig. 3. The primary components are the

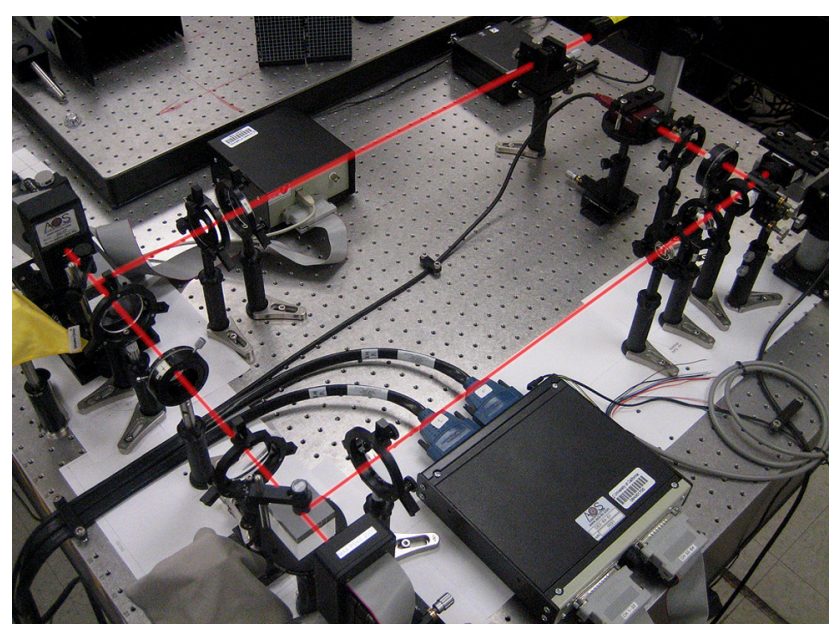

Fig. 2 Photograph of the experiment. laser source, two membrane deformable mirrors (DM), a Shack-Hartmann wavefront sensor, and target camera.

The illumination source in the experiment is a $0.8 \mathrm{~mW}$, continuous wave, helium-neon laser with a wavelength of $634 \mathrm{~nm}$. After exiting a spatial filter, the beam is expanded to approximately $20 \mathrm{~mm}$ in diameter and directed toward DM1, a membrane deformable mirror (DM) with 31 actuators, which alters the optical path difference (OPD) of the beam. A set of relay lenses image the beam onto DM2, a second membrane deformable mirror with 61 actuators. The beam is then split, with one branch directed to a wavefront sensor, and the other focused onto a target camera representing a far-field intensity pattern.

The two DM are manufactured by Active Optical Systems. Each DM has electrostatic actuators arranged in a hexagonal grid, with a maximum throw of approximately $10 \mu \mathrm{m}$. The mirror DM1 is the control actuator in the AO system, and DM2 is the disturbance actuator that puts the wavefront aberrations onto the beam, after the original aero-optical data is mapped to the geometry of DM2 as discussed in Sec. 3.2.

The drive electronics for the DM provide 8-bit resolution for the actuator voltages, which take integer values between 0 and 255. The displacement of each DM actuator is linearly proportional to the square of the applied actuator voltage, thus for the control loops, the actuator voltages are parameterized as

$$
v=\operatorname{round}\left(\sqrt{c+v_{b}^{2}}\right), \quad v_{b}=180 \simeq \sqrt{255^{2} / 2},
$$

where $v$ is the voltage to the DM actuator, $c$ is a control command and $v_{b}$ is an applied bias voltage. The bias $v_{b}$, which is the same for all actuators, produces a concave shape on the DM that can be corrected by adjusting subsequent imaging optics. Each command $c$ takes values in the interval $-v_{b}^{2} \leq c \leq v_{b}^{2}$. If the quantization error due to the rounding is neglected, then Eq. (25) yields an affine relationship between control commands and actuator displacements.

Wavefront measurements are provided by a CMOS Shack-Hartmann wavefront sensor, also manufactured by Active Optical Systems. The image plane of the sensor is divided into $12 \times 12$ subaperture grid, where each subaperture contains the focal point of a single lenslet in an $11 \times 11$ grid of pixels. The wavefront sensor output is a slope vector $y_{0}$ of dimension 288, containing the vertical and horizontal wavefront slopes over each of the 144 subapertures; this slope vector is used directly for control as discussed in Sec. 4 . For performance analysis, a $12 \times 12$ wavefront image $\phi$ with zero mean was generated by a zonal least-squares reconstructor matrix $E_{\phi}$, which was computed according to a well known method. ${ }^{26}$ Thus the vectorized wavefront image $\phi$ is constructed by

$\phi=E_{\phi} y_{0}$

A PC processes the wavefront sensor measurements, runs the control loops and drives both DMs. The experiment operates at a sample rate of approximately $40 \mathrm{~Hz}$ due to limitations of the hardware used; however, the $16 \mathrm{kHz}$ frame rate at which the aero-optical wavefronts were collected determines the spatial and temporal statistics of the open-loop and closed-loop wavefront sequences. Therefore, 


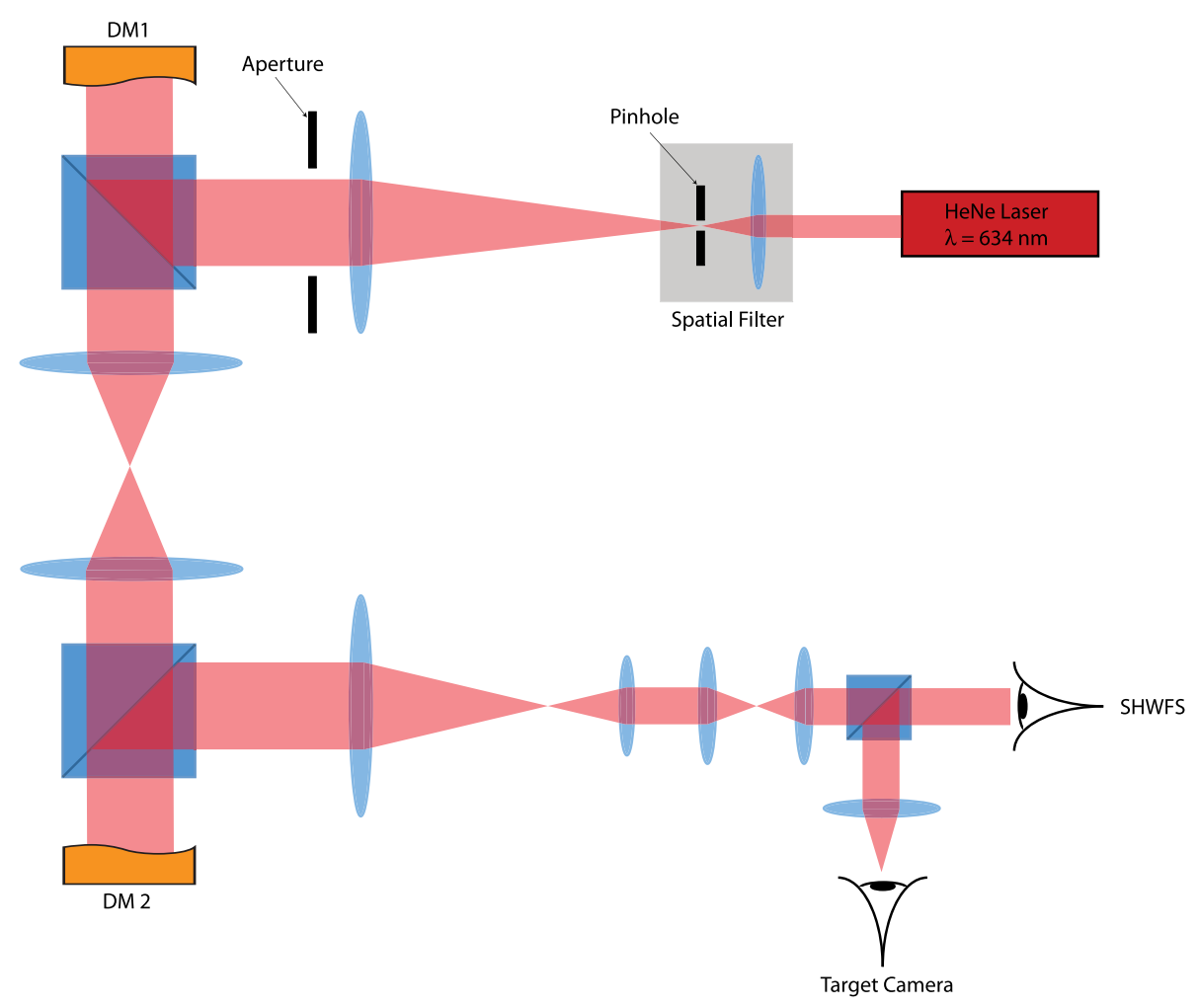

Fig. 3 Optical layout. The primary components are the laser source, two membrane deformable mirrors (DM1 and DM2), a Shack-Hartmann wavefront sensor (SHWFS), and target camera.

the performance of the control loops, the measured wavefront errors and the target camera images should be the same as if the experiment were running at the $16 \mathrm{KHz}$ frame rate.

\subsection{Aero-Optical Wavefront Disturbances in the AO Experiment}

The disturbance wavefronts used in the experiment came from Notre Dame's Airborne Aero-Optics Laboratory (AAOL). ${ }^{18}$ The original aero-optical wavefronts were produced by turbulence over a flat-windowed turret ${ }^{18,27-29}$ during a flight test in which a continuous-wave laser was transmitted between two planes flying in a constant formation at an altitude of $4570 \mathrm{~m}$. The planes were separated by approximately $50 \mathrm{~m}$ to ensure aero-optical turbulence was the primary source of wavefront aberrations. Other significant parameters for the flight test are give in Table 1.

While the predominant source of wavefront aberrations in the AAOL data is the turbulent boundary layer around the turret, some small electronic noise likely is present in the data. A detailed analysis of the statistics of the AAOL data has been published. ${ }^{18}$ Since the control loops in this paper attempt to minimize the total phase error that would be encountered in similar airborne environments, no attempts were made to distinguish between the sources of the measured turbulence.

For the $\mathrm{AO}$ experiment in this paper, a sequence of 8000 disturbance command vectors $c_{2}$ for the 61-actuator mirror DM2 was generated by mapping a sequence of 8000 aerooptical wavefronts from AAOL to the geometry of the experiment here. Details of this procedure are discussed in other publications. ${ }^{16,17}$ This mapping was done with the
Table 1 Experimental details for the Notre Dame AAOL data set.

\begin{tabular}{lc}
\hline \hline & \\
Turret azimuthal angle & $119 \mathrm{deg}$ \\
Turret elevation angle & $57 \mathrm{deg}$ \\
Free stream Mach & 0.36 \\
Altitude & $4570 \mathrm{~m}$ \\
Target distance & $50 \mathrm{~m}$ \\
Aperture size & $10.1 \mathrm{~cm}$ \\
Sampling rate & $16 \mathrm{kHz}$ \\
\hline \hline
\end{tabular}

goal of replicating the statistics of the aero-optical wavefront aberrations as closely as possible. The temporal statistics dictated by the $16 \mathrm{KHz}$ frame rate of the original AAOL data was maintained in the final wavefront disturbance sequence applied in the experiment here, even though the hardware limited the frame rate for the experiment to $40 \mathrm{~Hz}$.

\section{Control Problem for Adaptive Optics}

\subsection{Control Model of the AO Experiment}

The block diagram in Fig. 4 shows the signals and control loops for the AO problem. The $z^{-1}$ block indicates that the AO system has a one-sample loop delay, which is the overall latency due to deformable mirror response and wavefront sensor measurement read out. 


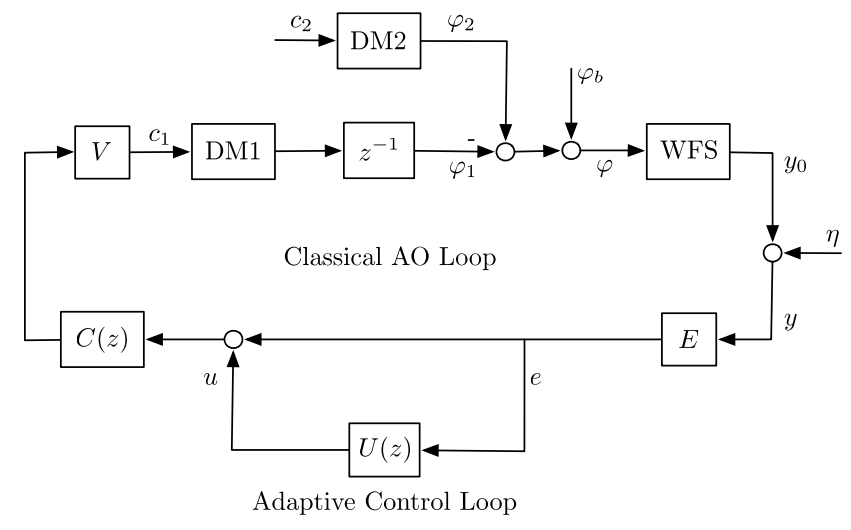

Fig. 4 Block diagram of the adaptive optics experiment.

The physical wavefront error that reaches the wavefront sensor is

$\varphi=\varphi_{b}+\varphi_{2}-\varphi_{1}$

where $\varphi_{b}$ is a static bias due to deformable mirror bias and optical misalignment, $\varphi_{2}$ is the wavefront disturbance added to the beam by the disturbance mirror DM2, and $\varphi_{1}$ is the phase correction provided by the control mirror DM1. For control design, the portion of the block diagram between control and disturbance commands $c_{1}$ and $c_{2}$ and the slope vector $y_{0}$ is modeled by

$y_{0}=y_{b}+\Gamma_{2} c_{2}-z^{-1} \Gamma_{1} c_{1}$,

where $y_{b}$ is the contribution to the slope vector from the wavefront bias $\varphi_{b}$, the vectors $c_{1}$ and $c_{2}$ are the command vectors to DM1 and DM2, respectively, and $\Gamma_{1}$ and $\Gamma_{2}$ are the corresponding poke matrices mapping actuator command vectors to slope vectors. The poke matrix $\Gamma_{2}$ is not used in either designing or implementing any of the controllers. Both $\Gamma_{1}$ and $\Gamma_{2}$ were identified by a least-squares fit to data as described in. ${ }^{16,17}$ Although significant nonlinearities exist in the experiment, the system is tuned to remain in an approximately linear regime.

The vector signal $\eta$ in Fig. 4 represents sensor noise added to the WFS vector to test the effect of measurement noise on the control loops. As shown in Fig. 4, the slope vector given to the control loops is

$y=y_{0}+\eta$.

A set of frequency-weighted DM modes that serve as control channels in all of the control loops. Figure 5 shows images of these modes. The frequency-weight modes have two important properties: orthogonality with respect to the actuator influence functions of DM1, and increasing spatial frequency with mode number. Since DM1 has 31 actuators, there are 31 modes. The modes are constructed for the particular geometry and actuator influence functions of DM1 by solving a certain eigenvalue problem involving the actuator influence functions of DM1. Details of this construction are given elsewhere. ${ }^{5}$

The columns of the matrix $V$ in Fig. 4 are the vectorized DM modes used in the control loops. The modal reconstructor matrix $E$, which maps the slope vector to modal coordinates, is the pseudo inverse of the modal poke matrix $\Gamma_{1} V$. It follows that

$E \Gamma_{1} V=I$

The number of columns in $V$ and rows in $E$ equals the number of DM modes used in the control loops. The vector signal

$e=E y$

represents the projection of the wavefront error onto the DM modes used in the control loops. Because of Eq. (30), the modal control channels are uncoupled when only the classical AO loop is closed. Hence, when either of the adaptive control loops are closed, each element of the control command vector $u$ affects only the corresponding modal channel in $e$. However, the adaptive controllers couple the

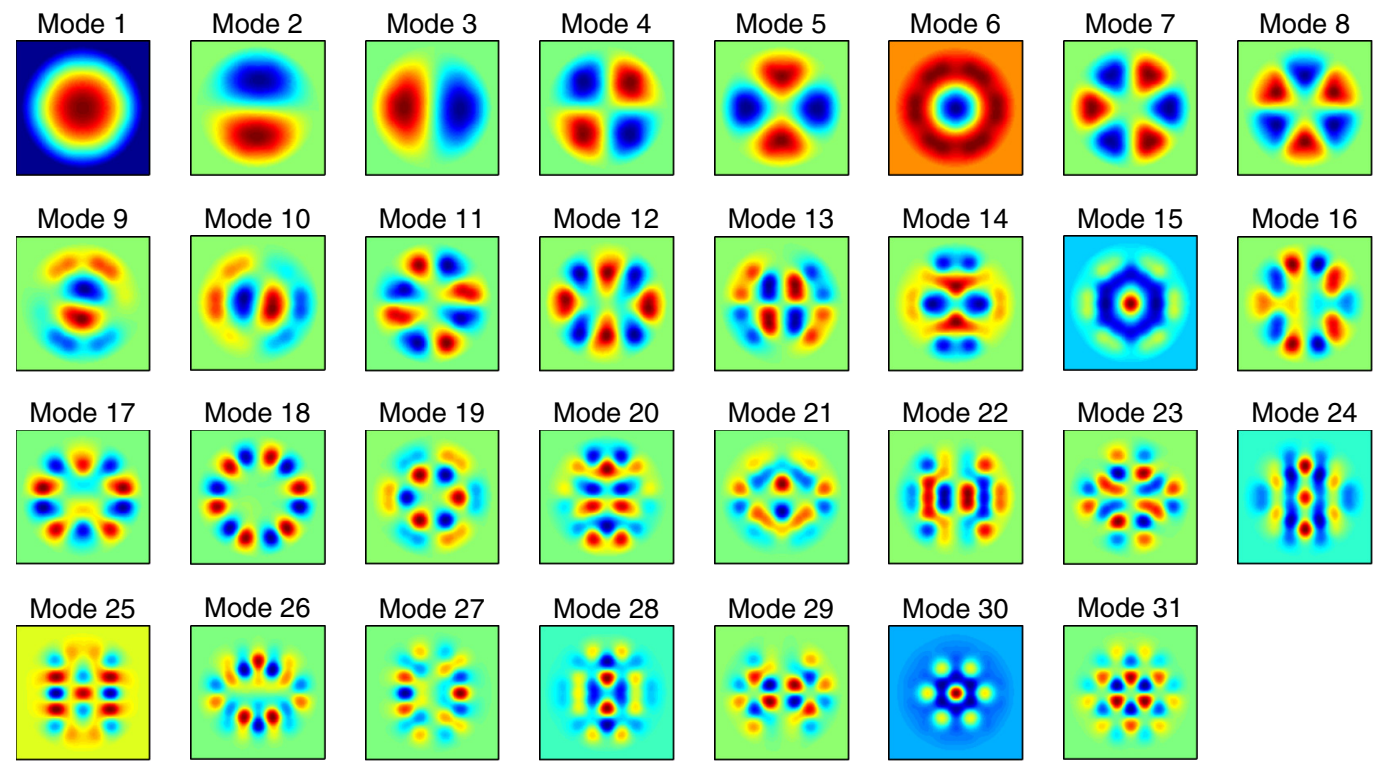

Fig. 5 Images of the frequency-weighted DM modes for DM1. 
channels by using sensor data from all channels used for control to determine each modal control command in $u$.

In the experiment, the DM actuators often saturate when all of of the modal control channels are used. This is due partly to long term drift in the DM characteristics, resulting from the sensitivity of membrane DM to environmental effects such as temperature. ${ }^{30}$ Saturation occurs primarily when the higher-order DM modes were included in the control loops. Modal analysis ${ }^{17}$ shows that the dominant wavefront disturbance in the aero-optical used here lies in modes 1 through 15 . Hence, for the experiments reported in this paper, only the first $15 \mathrm{DM}$ modes were used in the control loops. Other experiments showed no performance improvements with more controlled modes.

\subsection{Classical AO Control Loop}

The classical AO control loop shown in Fig. 4 consists of the least squares reconstructor matrix $E$, the modal matrix $V$ and the low-pass digital filter

$C(z)=\beta \frac{z}{z-\alpha}$

with gain $\beta$ and positive real pole $\alpha$. In the AO system, $C(z)$ is a diagonal multi-input-multi-output (MIMO) transfer function with each diagonal element equal to the scalar transfer function in Eq. (32). The controller $U(z)$ in Fig. 4, which augments the classical AO loop, is either the recedinghorizon adaptive controller described in Sec. 2 or the minimum-variance adaptive controller described in. ${ }^{4,5}$

As in Sec. 2, the true transfer function from the control command $u$ to the output error $e$ with only the classical AO loop closed is denoted by $G(z)$. The plant model $\hat{G}(z)$ used by the adaptive controllers is

$\hat{G}(z)=\frac{-\beta}{z+\beta-\alpha}$,

which is derived is derived from Fig. 4 with $u=0$ and with $C(z)$ given by Eq. (32). Because the signals $u$ and $e$ both are in DM modal coordinates and Eq. (30) implies that the plant model $\hat{G}(z)$ should be interpreted as a diagonal transfer matrix with each diagonal term given by Eq. (33). If there is no actuator saturation or quantization and the identified poke matrix $\Gamma_{1}$ accurately represents the mapping from DM1 commands to the wavefront sensor measurements, then

$$
G(z)=\hat{G}(z)
$$

When only the classical AO loop closed and Eq. (34) holds, the transfer function from the effect of the disturbance $\varphi_{2}$ on an individual controlled DM mode to the wavefront error for the same mode is the sensitivity transfer function, or error-rejection function,

$$
S(z)=\frac{z-\alpha}{z+\beta-\alpha} .
$$

For the experiments reported in Sec. 5, the pole in $C(z)$ was chosen as $\alpha=0.95$ to prevent integrator wind up and saturation of the actuator commands to DM1. The gain in $C(z)$ was $\beta=0.3$. Choosing $\beta$ involves a tradeoff between maximizing disturbance-rejection bandwidth and amplifying high frequency noise. Bode plots of the sensitivity transfer function $S(z)$ show that gains between $\beta=0.3$ and $\beta=0.5$ approximately maximize the disturbance rejection bandwidth without significantly amplifying high frequency noise. ${ }^{17}$ Experimental results for several choices of $\alpha$ and $\beta$ have shown that, when the DM1 commands do not saturate and closed-loop stability is maintained, the steady-state performance of the adaptive controllers indeed are largely independent of $\alpha$ and $\beta$.

The disturbance signal $w$ in Figs. 1 and 4 represents the projection onto the controlled DM modes of the residual wavefront error with only the classical AO loop closed (i.e., with $u=0$ ). The receding-horizon and minimum-variance adaptive controllers attempt to correct the portion of the wavefront disturbance represented by $w$. If $G(z)=\hat{G}(z)$, then

$w=S(z) E\left(y_{2}+y_{b}+\eta\right)$,

where $y_{2}$ and $y_{b}$ are the contributions to the open-loop slope vector from the disturbance and bias wavefronts $\varphi_{2}$ and $\varphi_{b}$, respectively, and $\eta$ is the sensor noise.

The plant model $\hat{G}(z)$, which is used by both adaptive controllers in this paper, does not include DM dynamics because any DM dynamics was negligible in the experiments reported here. The plant model used by the minimumvariance adaptive controller can include DM dynamics but only if the model of the DM dynamics does not couple the control channels. This can be a severe restriction in applications. As the equations in Sec. 2 show, the receding-horizon adaptive controller can include any finite-dimensional LTI model of DM dynamics, including models in which the control channels are coupled.

\section{Experimental Results}

\subsection{Description of Four Experiments}

This section presents results from four experiments to compare the performance of the receding-horizon adaptive controller introduced in this paper, the minimum-variance adaptive controller used in Ref. 5 and the classical AO loop alone. Of particular interest is the effectiveness of the frequency weighting in the receding-horizon performance index for mitigating the effect of high-frequency sensor noise. In each experiment, the deformable mirror DM2 placed the sequence of disturbance wavefronts onto the laser beam. Each experiment used the same 8000-frame aerooptical wavefront disturbance sequence, generated as described in Sec. 3. During the second half of each experiment (i.e., the last 4000 frames), the wavefront sensor noise described below was added to the measured wavefront sensor vector. The experiments were identical except for the control method used to compensate the wavefront disturbance.

Experiment 1: Open-loop. The control command $c_{1}$ to DM1 was zero, but disturbances were added to DM2 (i.e., $c_{2} \neq 0$ ).

Experiment 2: Classical AO only. The classical AO loop, described in Sec. 4, was closed (i.e., $u=0$ ).

Experiment 3: Receding-horizon (RH) adaptive control. Both the classical and receding-horizon controllers were closed. At $t=0$ the 
adaptive prediction filter contained in the receding-horizon controller (with $N=10$ ) began a period of 500 learning steps. The receding-horizon control loop began generating commands at $t=300$.

Experiment 4: Minimum-variance (MV) adaptive control. Both the classical and minimum-variance adaptive controllers were closed. At $t=0$ the adaptive filter $F(z)$ (with $N=10$ ) began a period of 500 learning steps. The adaptive control loop began generating control commands at $t=300$.

\subsection{Sensor Noise}

Each experiment used the same sensor noise sequence $\eta$, which was added to the wavefront sensor measurement as shown in the block diagram in Fig. 4. During the time interval $t \in[0,4000]$, no sensor noise was added to the WFS measurements (i.e., $\eta=0$ ). During the interval $t \in[4001,8000]$, the sequence $\eta$ was a zero-mean sequence produced by passing a white-noise sequence through a high-pass Butterworth filter with a cut-off frequency of $5600 \mathrm{~Hz}$. The added sensor noise had high-frequency temporal statistics with the power spectral density (PSD) shown in Fig. 6. A scalar sequence with this PSD was added to each element of the measured slope vector. For the results presented here, the noise sequences added to different elements of the slope vector were uncorrelated, so that the sensor noise had white spatial statistics. Coloring the spatial statistics of the noise made little difference in the experiment.

For the period $t \in[4000,8000]$, the signal-to-noise ratios for the horizontal and vertical slope vectors were

$$
\mathrm{SNR}_{\mathrm{hor}}=\frac{\left\|y_{0 \text { hor }}\right\|}{\|\eta\|}=2.09, \quad \mathrm{SNR}_{\mathrm{vert}}=\frac{\left\|y_{0 \text { vert }}\right\|}{\|\eta\|}=1.58
$$

where $y_{0 \text { hor }}$ and $y_{0 \text { vert }}$ are the halves of the slope vector $y_{0}$ containing the horizontal and vertical slope measurements, respectively, and $\|\cdot\|$ denotes the root-mean-square

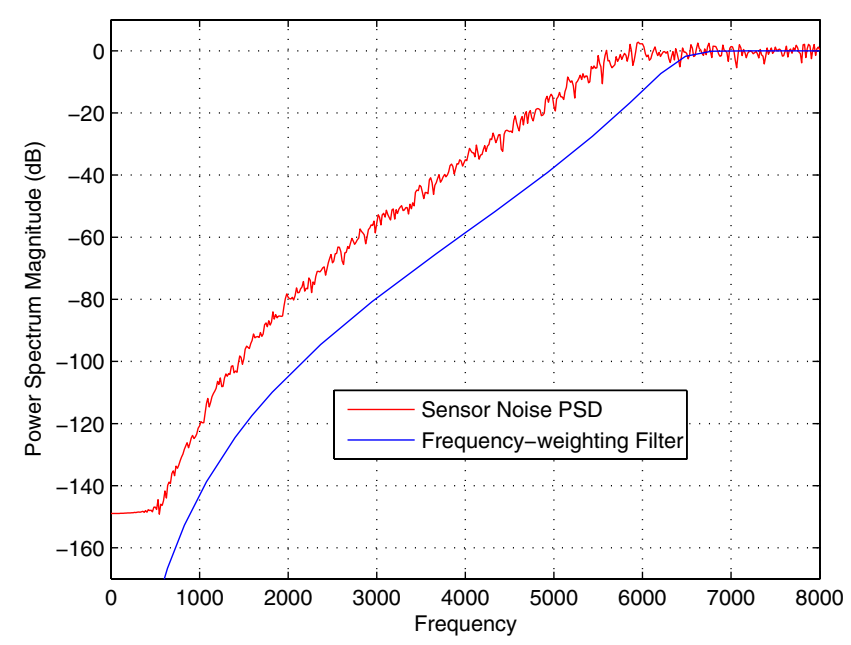

Fig. 6 Power spectral density for the sensor noise sequence $\eta$, added for time steps 4001 to 8000 ; also, Bode magnitude plot of frequencyweighting filter for receding-horizon adaptive control design.
(RMS) over space and time. (To facilitate comparison with the frequency-weighting filter, the sensor noise PSD in Fig. 6 was scaled to have maximum value $0 \mathrm{~dB}$.) The noise sequence $\eta$ was the same for each of the four experiments, and $\eta$ had the same RMS value for each direction. The larger SNR for the horizontal direction likely resulted from the fact that the average horizontal flow velocity was approximately twice the average vertical flow velocity, so that the turbulence that produced the aero-optical effects produced larger slopes in the dominant flow direction.

High-frequency sensor noise was used for the results presented in this paper because disturbance-rejecting adaptive controllers typically are more sensitive to high-frequency noise than to white noise. This is due to the fact that the adaptive filter in the a disturbance-rejecting adaptive controller predicts the disturbance and therefore has high gain at high frequencies. In presence of sensor noise, the high-frequency gain of the adaptive filter without frequency weighting becomes even larger as the filter attempts to predict the highfrequency part of the noisy error signal. As a result, the adaptive controller amplifies the high-frequency noise and passes it through to the actuators. The effect is most severe when the total noise power is concentrated at high frequencies, and that is why high-frequency noise was chosen for the results presented here.

White sensor noise was used in some experiments otherwise like the ones for which results are presented here. The results for white sensor noise were qualitatively similar to the results here for high-frequency sensor noise but quantitatively less dramatic. White sensor noise with RMS values roughly equal to those of the high-frequency noise used here degraded the performance of both adaptive controllers but not as much as the high-frequency noise degraded the performance. In the presence of white sensor noise, the frequency-weighted receding-horizon adaptive controller again performed better than the minimum-variance adaptive controller. For white sensor noise, the adaptive controller was designed with the same high-pass frequency-weighting filter used here because the high-frequency gain of the adaptive prediction filter causes most of sensitivity to noise, whether the noise is white or predominantly high-frequency.

\subsection{Adaptive Control Design}

To mitigate the effect of the high-frequency sensor noise, the receding-horizon adaptive controller included frequency weighting on the adaptive control command signal as described in Sec 2.3. The frequency-weighting filter was a sixth-order high-pass Butterworth filter with cut-off frequency of $6400 \mathrm{~Hz}$. The Bode magnitude plot of this filter is shown in Fig. 6. The frequency-weighting filter penalized the control signal at high frequencies to prevent it from amplifying the sensor noise and passing it through to the closed-loop wavefront. It seems reasonable to assume that an approximate sensor noise bandwidth is known in applications, but not the exact bandwidth. Thus the cut-off frequency of the weighting filter was chosen to be $800 \mathrm{~Hz}$ higher than the cut-off frequency for the sensor noise.

For the receding-horizon adaptive controller, the horizon length in the performance index $J_{h}$ was $h=20$ and the adaptive FIR lattice filter $F(z)$ had 10 taps. The control weighting in $J_{h}$ was $R=10^{-4}$. The minimum-variance adaptive controller uses an adaptive FIR lattice filter to predict residual 


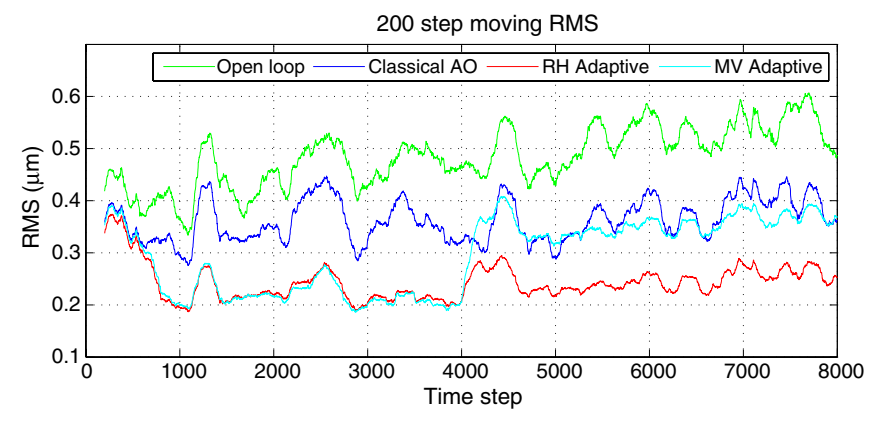

Fig. 7 RMS value of the wavefront error computed over space and time for a 200 -frame moving window. High-frequency sensor noise was added to the WFS measurement during the last 4000 time steps.

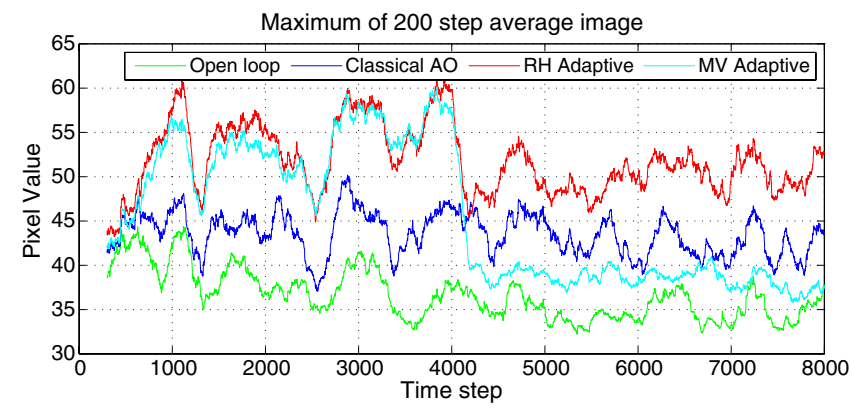

Fig. 8 Maximum intensity in the average target camera image for the 200-frame moving window. High-frequency sensor noise was added to the WFS measurement during the last 4000 time steps. wavefronts. ${ }^{5,17}$ This adaptive filter also had 10 taps in the experiments reported here.

\subsection{Performance in Terms of Wavefront Error and Target Camera Image}

Figures 7-10 illustrate the effectiveness of the control loops for reducing the total wavefront error measured by the wavefront sensor, and increasing the intensity measured by the target camera. The plot in Fig. 7 shows the RMS value of the reconstructed wavefront errors, computed over the $12 \times 12$ wavefront sensor grid discussed in Sec. 3.1 and a 200-frame moving window. The plot in Fig. 8 shows peak intensities of average target camera images computed over the 200-frame moving window. Figs. 9 and 10 show average target camera images for the portions of the experiments with and without sensor noise added.

For wavefront sensor results, the wavefront images were reconstructed on the $12 \times 12$ wavefront sensor grid by Eq. (26) as discussed in Sec. 3.1. It should be emphasized that the wavefront errors used for performance analysis were reconstructed from the measured wavefront sensor vector $y_{0}$. The only way in which this wavefront sensor vector is affected by the sensor noise $\eta$ is that the control loops can pass it through to the control actuator DM1.

As shown in Fig. 7, during the first 4000 time steps, both adaptive controllers reduced the wavefront error significantly as compared to the classical AO loop once the respective adaptive control loops were closed. The two adaptive controllers should perform similarly when there is little or no sensor noise. During the second 4000 time steps, the added sensor noise caused the minimum-variance adaptive controller to reduce the wavefront error only minimally relative to
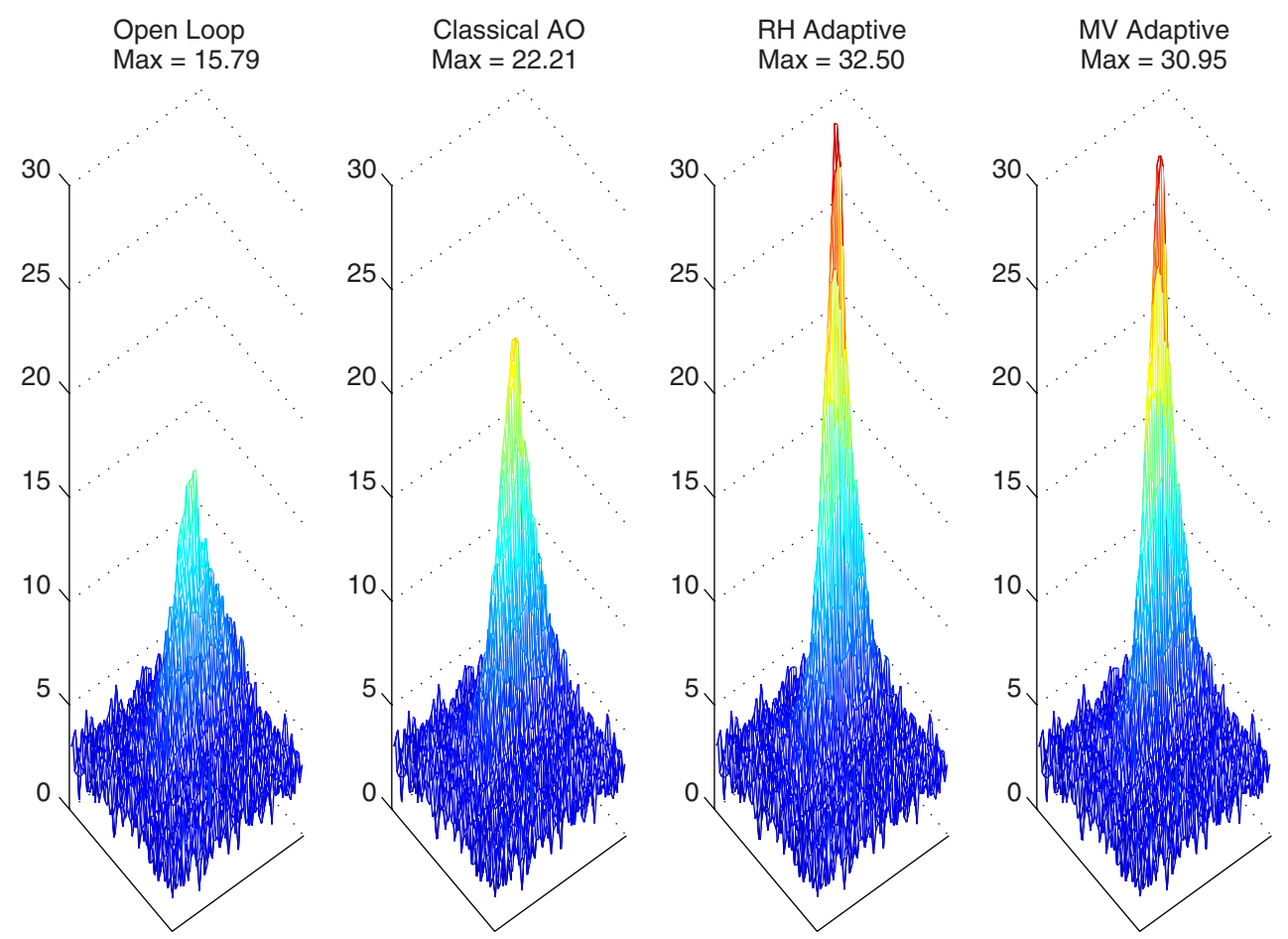

Fig. 9 Mean target camera images for time steps 300 to 4000 (no added sensor noise). Target camera images are $60 \times 60$ pixels, with pixel pitch size $=6.7 \mu \mathrm{m}$. 

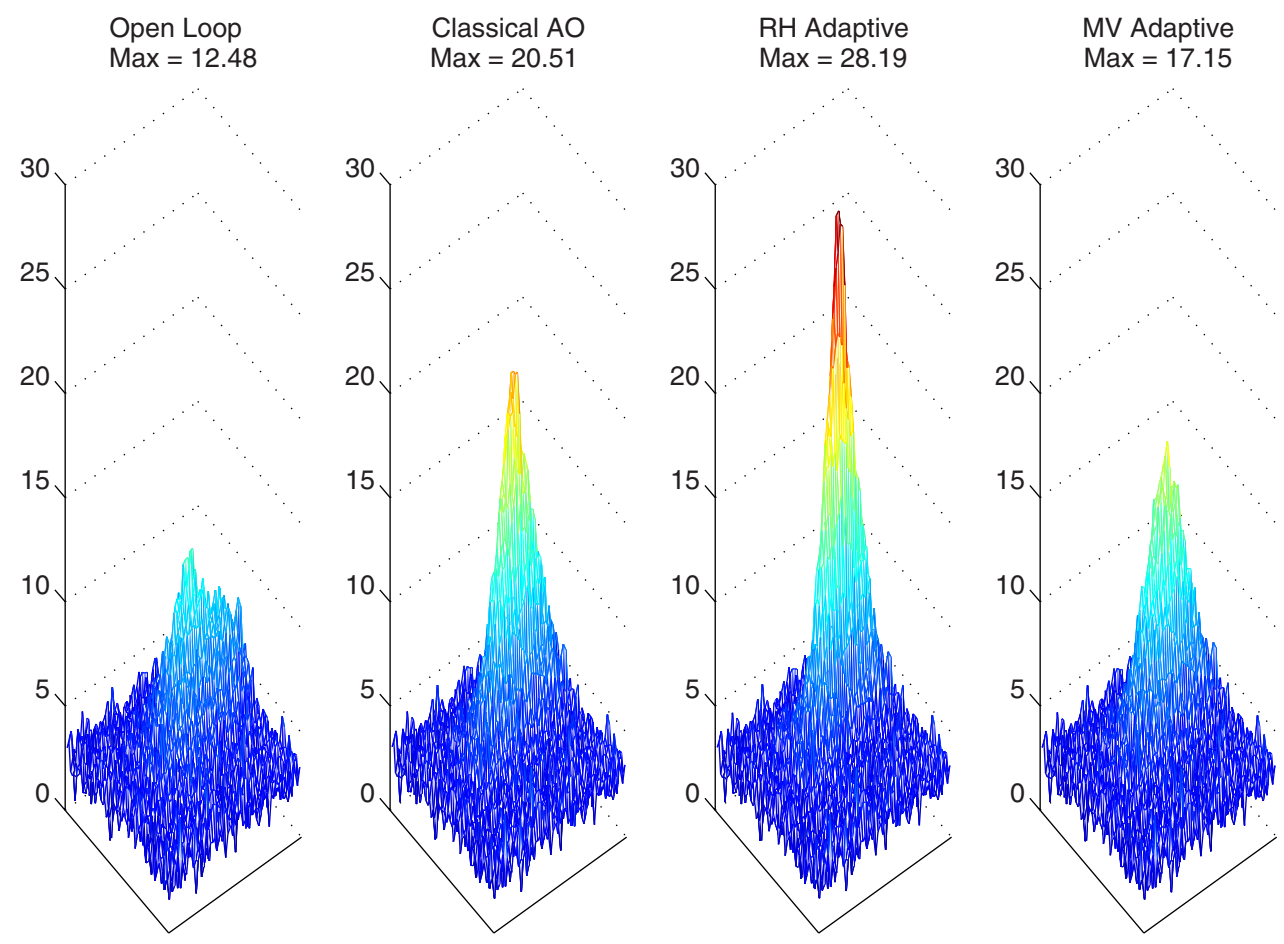

Fig. 10 Mean target camera images for time steps 4300 to 8000 (sensor noise added to WFS vector). Target camera images are $60 \times 60$ pixels, with pixel pitch size $=6.7 \mu \mathrm{m}$.

the classical AO loop, whereas the receding-horizon adaptive controller maintained a large reduction in the wavefront error relative to the classical AO loop.

Results from the target camera shown in Fig. 8 and the mesh plots in Figs. 9 and 10 show performance characteristics seen in the wavefront error. The performance of the receding-horizon and minimum-variance adaptive controllers were very similar in the first half of the experiments after the adaptive loops were closed. However, Figs. 8 and 10 show that the performance of the minimum-variance adaptive controller deteriorated severely when sensor noise was added after time step 4000, whereas the reduced highfrequency amplification of the receding-horizon adaptive controller allowed it to continue to achieve significant performance improvement in the maximum target camera intensity, corresponding to the improvement in wavefront error shown in Fig. 7.

The jitter of the location of the peak intensity in the target camera image provides another useful comparison of the performance of the different control loops. The scatter plots in Figs. 11 and 12 show the frequency with which the maximum intensity appeared in each pixel of the target camera image. For the 4000 time steps with no sensor noise, both adaptive controllers significantly reduced the jitter of the location of the peak intensity. Once sensor noise was added during the second 4000 time steps, the minimum-variance adaptive controller reduced the jitter much less than did the receding-horizon adaptive controller.

Comparison of the target camera image results in Figs. 7-10 for the classical AO loop and the minimumvariance adaptive controller indicate that, with the added sensor noise, the deterioration in the average target camera images produced by the minimum-variance adaptive controller is even worse than the results for the wavefront errors
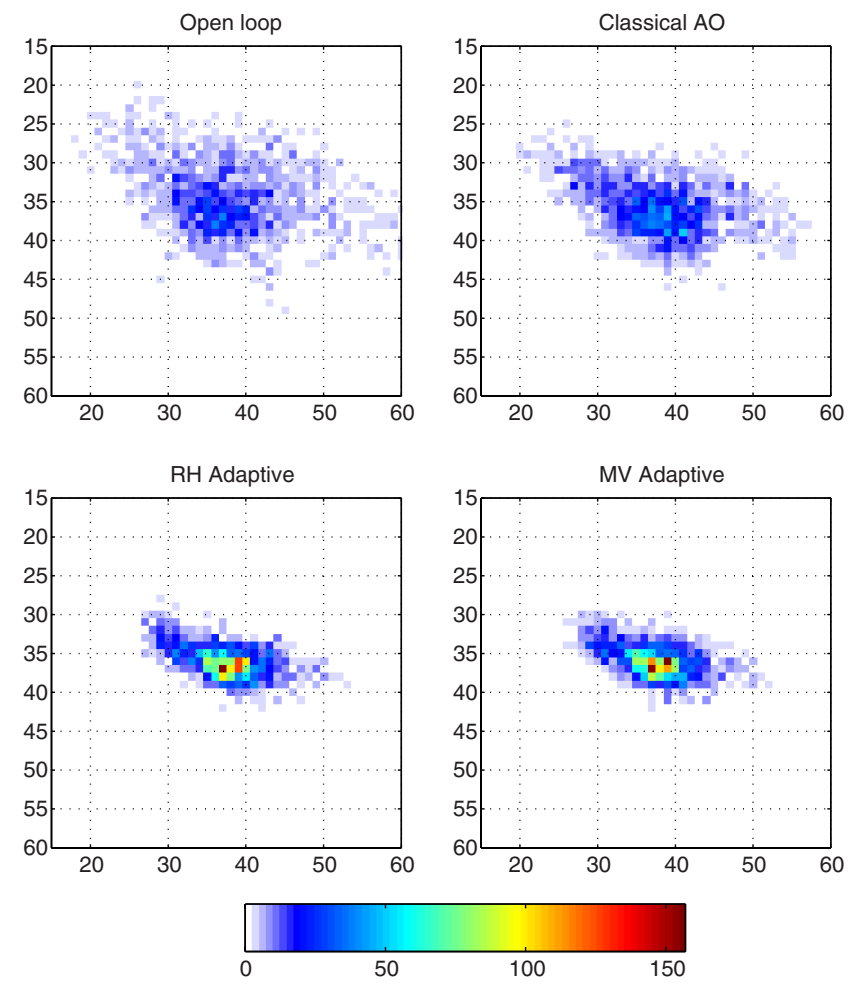

Fig. 11 Scatter plots for the location of the maximum target camera intensity for time steps 300 to 4000 (no added sensor noise).

would suggest. This likely results from the severe highfrequency disturbance that the minimum-variance adaptive controller adds to the laser beam in DM modes 1 and 2, especially since mode 1 is focus. That the minimum-variance controller passes high-frequency noise to the closed-loop 

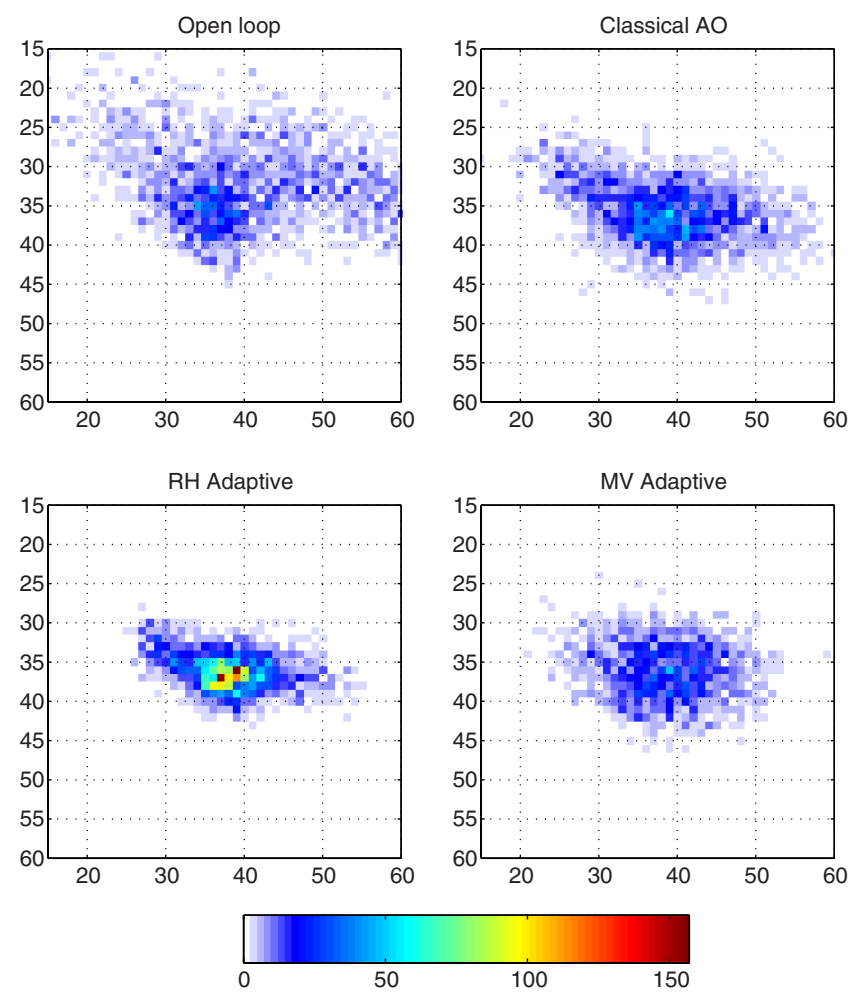

Fig. 12 Scatter plots for the location of the maximum target camera intensity for time steps 4300 to 8000 (sensor noise added to WFS vector).
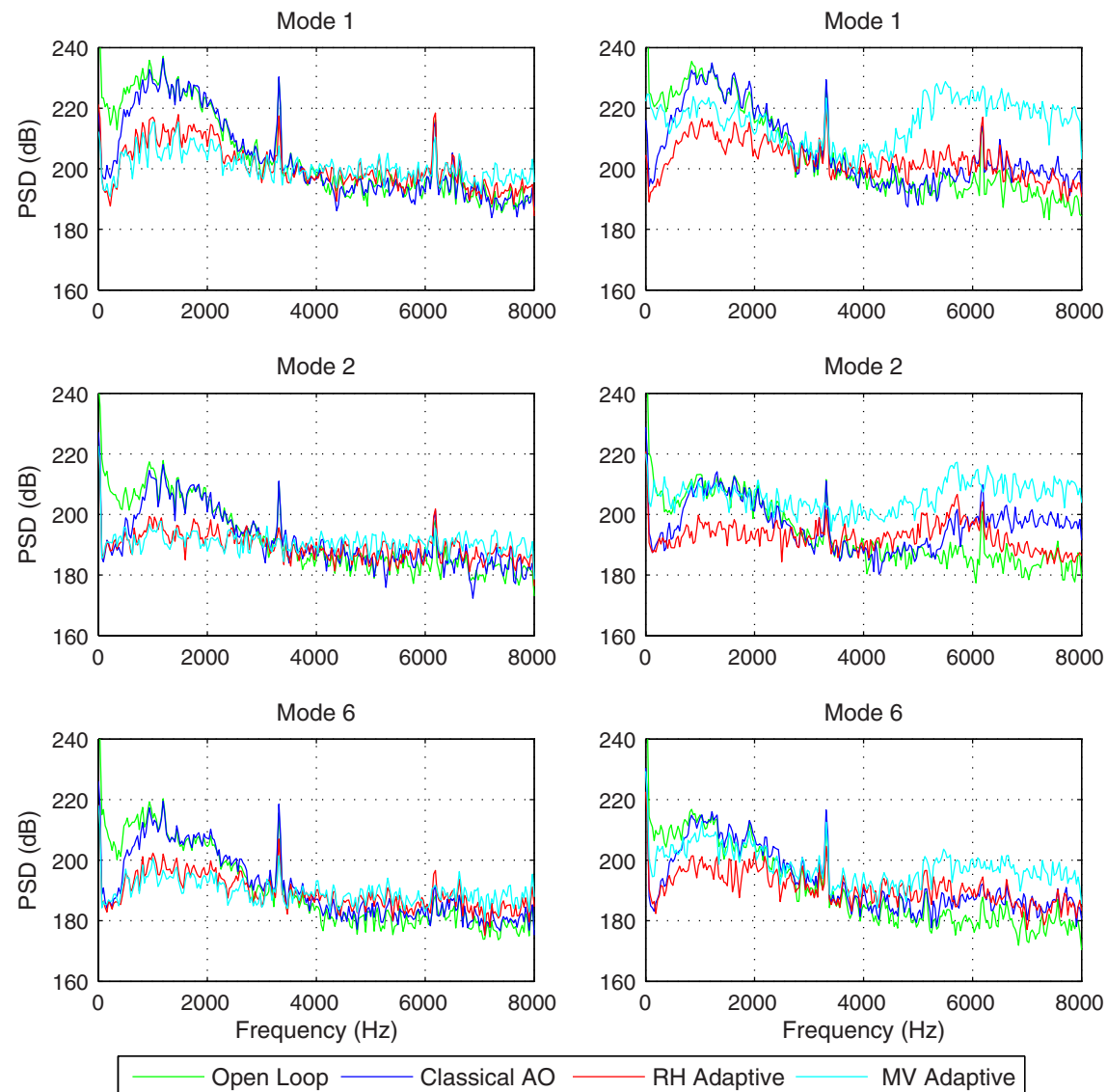

- RH Adaptive — MV Adaptive

Fig. 13 Power spectral densities of the modal time series for the three DM modes with largest power. Left: for time steps 300 to 4000 (no added sensor noise). Right: for time steps 4300 to 8000 (sensor noise added to WFS vector). 

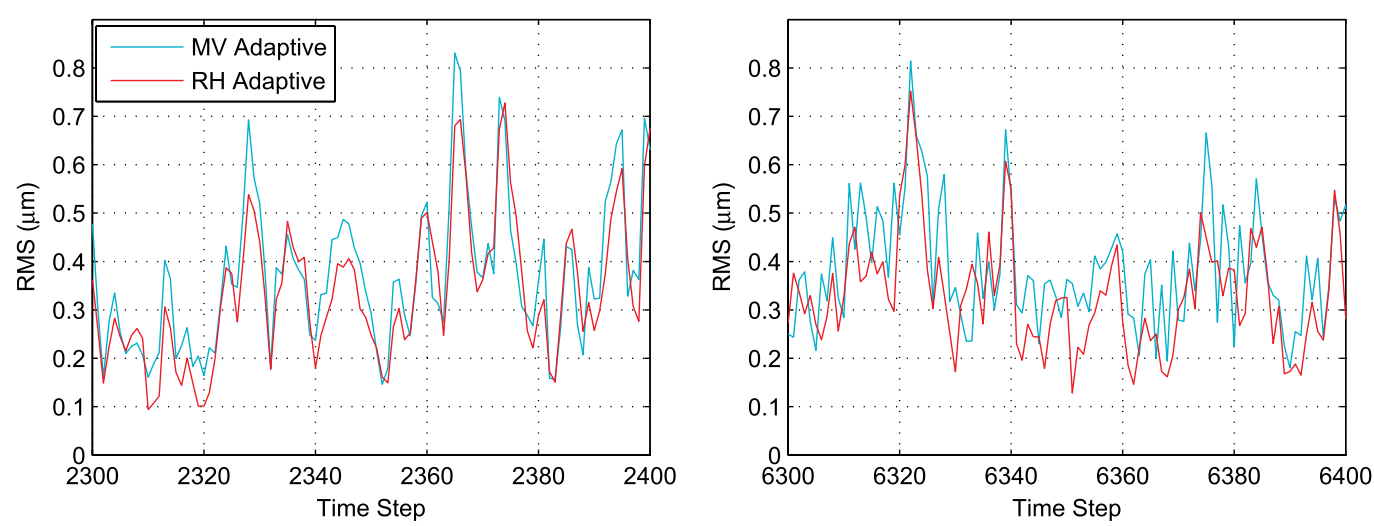

Fig. 14 Spatial RMS values of the wavefront corrections produced by DM1, plotted for representative time intervals. Left: for time steps 300 to 4000 (no added sensor noise). Right: for time steps 4300 to 8000 (sensor noise added to WFS vector).

However for the interval $t \in[4000,8000]$, during which the high-frequency sensor noise was added, the PSDs on the right in Fig. 13 show that the effectiveness of the adaptive controllers changed. The minimum-variance adaptive controller significantly amplified the high frequency sensor noise. The receding-horizon controller amplified high frequency noise and disturbance somewhat relative to the classical AO loop in the bandwidth between 4000 and $6000 \mathrm{~Hz}$ approximately, but the frequency weighting in the receding-horizon controller prevented the egregious noise amplification produced by the minimum-variance adaptive controller. Also, the PSDs show that the added sensor noise greatly reduced the effectiveness of minimumvariance adaptive controller in the frequency range below $2500 \mathrm{~Hz}$, even though most of the added noise was above $4000 \mathrm{~Hz}$.

\subsection{Magnitudes of Control Commands}

Finally, it is noteworthy to compare the magnitudes of the control commands to the deformable mirror DM1 for the two adaptive controllers and the resulting wavefront corrections that DM1 adds to the laser beam. These wavefront corrections are denoted by $\varphi_{1}$ in Fig. 4 . Since the wavefront corrections were not directly observable, they are reconstructed as images on the $12 \times 12$ wavefront sensor grid discussed in Sec. 3.1. For each control command vector $c_{1}$, the corresponding wavefront correction is constructed as

$\phi_{1}=E_{\phi} \Gamma_{1} c_{1}$,

where $E_{\phi}$ is the least-squares wavefront reconstructor in Eq. (26) and $\Gamma_{1}$ is the poke matrix for DM1. Thus the wavefront corrections are reconstructed in the same way that the wavefront errors were constructed by Eq. (26) for Fig. 7 except that, whereas the measured slope vector $y_{0}$ was used in Eq. (26), the poke matrix $\Gamma_{1}$ is used in Eq. (39) to map a command vector $c_{1}$ to the corresponding slope vector.

For representative intervals, Fig. 14 shows the time series consisting of spatial RMS values of reconstructed wavefront corrections $\phi_{1}$ produced by the two adaptive controllers. Table 2 gives the RMS values over space and time of reconstructed wavefront corrections. (The RMS values in Table 2 are the RMS values of the curves plotted in Fig. 14 computed over the longer time intervals indicated.) Table 2 also gives the total number of saturated DM1 actuator commands for
Table 2 Numbers of saturated DM1 actuators and RMS values over time and space of the wavefront corrections produced by DM1, for time intervals $T_{1}=$ time steps 300 to 4000 and $T_{2}=$ time steps 4300 to 8000

\begin{tabular}{|c|c|c|c|c|}
\hline & \multirow{2}{*}{\multicolumn{2}{|c|}{$\begin{array}{c}\begin{array}{c}\text { Total } \\
\text { saturated }\end{array} \\
\text { Actuators }\end{array}$}} & \multirow{2}{*}{\multicolumn{2}{|c|}{$\begin{array}{l}\begin{array}{c}\text { DM1 RMS } \\
(\mu \mathrm{m})\end{array} \\
\text { Wavefronts }\end{array}$}} \\
\hline & & & & \\
\hline & $T_{1}$ & $T_{2}$ & $T_{1}$ & $T_{2}$ \\
\hline Receding horizon adaptive & 20 & 100 & 0.28 & 0.32 \\
\hline Minimum variance adaptive & 91 & 291 & 0.31 & 0.39 \\
\hline
\end{tabular}

the time interval between time steps 300 and 4000 and the time interval between time steps 4300 and 8000 .

As illustrated by Fig. 14 and Table 2, the receding-horizon adaptive controller used less control effort; i.e., the recedinghorizon controller produced smaller control commands and wavefront corrections than the minimum-variance adaptive controller. Yet in terms of reduced wavefront errors and sharper target camera images, the receding-horizon controller performs as least as well as the minimum-variance controller when there is no added sensor noise and much better than he minimum-variance controller in the presence of the added sensor noise.

\section{Conclusions}

The receding-horizon adaptive control scheme presented in this paper provides a natural and flexible way to include frequency weighting and uncertainty models in the design of the adaptive controller. The experimental results here demonstrate the effectiveness of frequency weighting in the receding-horizon adaptive controller for reducing sensitivity with respect to high-frequency sensor noise. As the closedloop wavefront errors and the target camera images show, the minimum-variance adaptive controller used previously in $\mathrm{AO}$ and used here for comparison passes the high-frequency sensor noise through to the optical wavefronts, thereby significantly degrading the performance of the AO system. The receding-horizon adaptive controller reduced wavefront errors and sharpened target camera images as least as well as the minimum-variance adaptive controller with no sensor 
noise and much better than the minimum-variance adaptive controller with sensor noise. Also, the receding-horizon adaptive controller achieved the improved performance with smaller control effort.

\section{Acknowledgments}

This work was supported by the High Energy Laser Joint Technology Office and the U.S. Office of Naval Research under Grant No. 00014 07-1-1063. The authors of this paper are indebted to the authors of Refs. 18, 27-29 for the aerooptical data and indispensable assistance with interpreting the data.

\section{References}

1. B. L. Ellerbroek and T. A. Rhoadarmer, "Real-time adaptive optimization of wavefront reconstruction algorithms for closed-loop adaptive optical systems," Proc. SPIE 3353, 1174-1185 (1998).

2. J. S. Gibson, C. C. Chang, and B. L. Ellerbroek, "Adaptive optics: wavefront correction by use of adaptive filtering and control," Appl. Opt. 39 (16), 2525-2538 (2000)

3. T. A. Rhoadarmer et al., "Adaptive control and filtering for closed-loop adaptive-optical wavefront reconstruction," Proc. SPIE 6306, 63060E (2006).

4. Y. T. Liu and J. S. Gibson, "Adaptive control in adaptive optics for directed-energy systems," Opt. Eng. 46(4), 046601 (2007).

5. S. Monirabbasi and J. S. Gibson, "Adaptive control in an adaptive optics experiment," J. Opt. Soc. Am. A 27(11), A84-A96 (2010).

6. C. Petit et al., "Off-axis adaptive optics with optimal control: experimental and numerical validation," Proc. SPIE 5903, 59030P (2005).

7. K. Hinnen, M. Verhaegen, and N. Doelman, "Exploiting the spatiotemporal correlation in adaptive optics using data-driven $\mathrm{H}_{2}$-optimal control," J. Opt. Soc. Am. A 24(6), 1714-1725 (2007).

8. K. Hinnen, M. Verhaegen, and N. Doelman, "A data-driven calH ${ }_{2}$-optimal control approach for adaptive optics," IEEE Trans. Control Syst. Technol. 16(3), 381-395 (2008).

9. C. Petit et al., "Linear quadratic gaussian control for adaptive optics and multiconjugate adaptive optics: experimental and numerical analysis," J. Opt. Soc. Am. A 26(6), 1307-1325 (2009).

10. R. Fraanje et al., "Fast reconstruction and prediction of frozen flow turbulence based on structured Kalman filtering," J. Opt. Soc. Am. A 27 (11), A235-A245 (2010).

11. A. Beghi, A. Cenedese, and A. Masiero, "A Markov-Random-Fieldbased approach to modeling and prediction of atmospheric turbulence," in 16th Mediterranean Conf. on Control and Automation, pp. 17351740, IEEE, Ajaccio, Corsica, France (2008).

12. A. Beghi, A. Cenedese, and A. Masiero, "Stochastic realization approach to the efficient simulation of phase screens," J. Opt. Soc. Am. A 25(2), 515-525 (2008)

13. A. Beghi, A. Cenedese, and A. Masiero, "Multiscale stochastic approach for phase screens synthesis," Appl. Opt. 50(21), 4124-4133 (2011).

14. J. Tesch et al., "Identification, prediction and control of aero optical wavefronts in laser beam propagation," Conf. on 41st AIAA Plasmadynamics and Lasers, AIAA, Honolulu, Hawaii (2011).

15. J. Tesch and J. S. Gibson, "Optimal and adaptive correction of aerooptical wavefronts in an adaptive optics experiment," Proc. SPIE 8165, 816502 (2011).

16. J. Tesch, "High-performance control and prediction for adaptive optics," PhD Thesis, University of California, Los Angeles (2011).

17. J. Tesch and S. Gibson, "Optimal and adaptive control of aero-optical wavefronts for adaptive optics," J. Opt. Soc. Am. A 29(8), 1625-1638 (2012).

18. C. Porter et al., "Flight measurements of aero-optical distortions from a flat-windowed turret on the airborne aero-optics laboratory (AAOL)," Conf. on 42nd AIAA Plasmadynamics and Lasers, AIAA, Honolulu, Hawaii (2011).

19. R. R. Bitmead, M. Gevers, and V. Wertz, Adaptive Optimal Control: The Thinking Mans GPC, Prentice-Hall, Upper Saddle River, New Jersey (1990).

20. E. Mosca, Optimal, Predictive and Adaptive Control, Prentice-Hall, Upper Saddle River, New Jersey (1995).

21. W. H. Kwon and S. Han, Receding Horizon Control, Springer-Verlag, London (2005).

22. N. O. P. Arancibia, S. Gibson, and T.-C. Tsao, "Frequency-weighted minimum-variance adaptive control of laser beam jitter," IEEE ASME Trans. Mechatron. 14(3), 337-348 (2009).

23. P. K. Orzechowski et al., "Optimal suppression of laser beam jitter by high-order RLS adaptive control," IEEE Trans. Control Syst. Technol. 16(2), 255-267 (2008).
24. S. B. Jiang and J. S. Gibson, "An unwindowed multichannel lattice filter with orthogonal channels," IEEE Trans. Signal Process. 43(12), 28312842 (1995)

25. A. H. Sayed, Fundamentals of Adaptive Filtering, John Wiley and Sons, Hoboken, New Jersey (2003).

26. W. H. Southwell, "Wave-front estimation from wave-front slope measurements," J. Opt. Soc. Am. 70(8), 998-1006 (1980).

27. S. Gordeyev and E. Jumper, "Fluid dynamics and aero-optics of turrets," Progr. Aerospace Sci. 46(8), 388-400 (2010).

28. S. Gordeyev et al., "Aero-optical environment around a cylindrical turret with a flat window," AIAA J. 49(2), 308-315 (2011).

29. M. Weng, A. Mani, and S. Gordeyev, "Physics and computation of aerooptics," Annu. Rev. Fluid Mech. 44(1), 299-321 (2012).

30. J. D. Mansell and B. Henderson, "Temporal and spatial characterization of polymer membrane deformable mirrors," Proc. SPIE 7466, 74660D (2009).

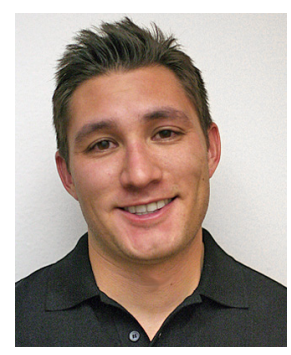

Jonathan Tesch is an engineer at the Jet Propulsion Laboratory in Pasadena, California working on control and prediction for adaptive optics systems. He received the BS degree from the University of California, Berkeley in 2006, and the MS and PhD degrees in 2007 and 2011 from the University of California, Los Angeles, all in mechanical engineering.

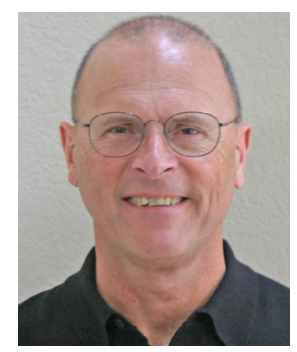

Steve Gibson received the BS degree in aerospace engineering, the MS degree in engineering mechanics, and the PhD degree in engineering mechanics from The University of Texas at Austin, Austin, in 1970, 1972, and 1975, respectively. He served on the faculties of the Aerospace Engineering and Engineering Mechanics Department, The University of Texas at Austin and the Engineering Science and Mechanics Department, Virginia Polytechnic Institute and State University. In 1977, he joined the faculty of the University of California, Lost Angeles (UCLA), where he currently is a professor of Mechanical and Aerospace Engineering. His research interests include control and identification of dynamical systems and adaptive filtering, with applications to identification and control of flexible structures, control of laser beams and adaptive optics, identification and control of micro inertial sensors, control of fluid flow and noise control. He has served as an associate editor for the SIAM Journal on Control and Optimization and for the IEEE Transactions on Automatic Control.

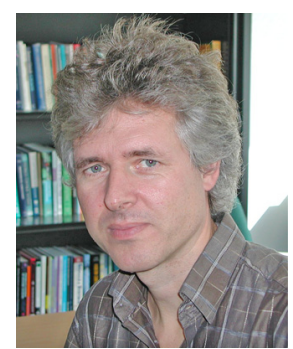

Michel Verhaegen received an engineering degree in aeronautics from the Delft University of Technology, The Netherlands, in August 1982, and the doctoral degree in applied sciences from the Catholic University Leuven, Belgium, in November 1985. From 1986 to 1988 he was affiliated with the NASA Ames research center in California. From 1989 to 1994 he was a research fellow of the Dutch Academy of Arts and Sciences, affiliated with the Network Theory Group of the Delft University of Technology. In the period 1994 to 1999 he was an associate professor of the control laboratory of the Delft University of Technology and became appointed as full professor at the faculty of Applied Physics of the university of Twente in the Netherlands in 1999. From 2001, he moved back to the University of Delft and is now a member of the Delft Center for Systems and Control. His main research interest is in the interdisciplinary domain of numerical linear algebra and system theory. In this field he has published over 120 papers in peer reviewed Journals. Current activities focus on new methodologies for identification, distributed, fault tolerant control and data driven controller design. 